\title{
REVIEW
}

\section{Emergency management of cardiac chest pain: a review}

\author{
K R Herren, K Mackway-Jones
}

Chest pain accounts for $2 \%-4 \%$ of all new attendances at emergency departments (ED) in the United Kingdom. ${ }^{12}$ Chest pain can be the presenting complaint in a myriad of disorders ranging from life threats such as acute myocardial infarction (AMI) to mild self limiting disorders such as muscle strain. Possible cardac chest pain can be viewed as a continuum, ranging from total global AMI to simple short lived angina. Within this spectrum lie the acute coronary syndromes with critical cardiac ischaemia and minimal myocardial damage.

Nationally over 129000 deaths a year are attributable to ischaemic heart disease. ${ }^{3}$ AMI case mortality is currently $45 \%$ with over $70 \%$ of these dying before they reach medical care. ${ }^{4}$ One in eight patients with unstable angina will infarct within two weeks without appropriate treatment. In the UK around 30\% of patients with chest pain are admitted and $70 \%$ discharged from the $\mathrm{ED}^{1}$ while in the United States $60 \%$ are admitted and $40 \%$ discharged. ${ }^{4}$ Despite such high admission rates $3 \%-4 \%$ of AMI are inadvertently discharged from US EDs. In the UK significantly fewer patients are admitted; while the number of missed AMIs is unknown, recent evidence suggests that some $6 \%$ of patients discharged from EDs may have prognostically significant myocardial damage. ${ }^{5}$

Mortality for patients with AMI differs greatly between admitted and discharged patients $\left(6 \%\right.$ versus 25\%). ${ }^{6}$ Missed AMI accounts for $20 \%$ of US emergency medicine related litigation dollars. ${ }^{7}$ Many interventions including drug therapy and surgery reduce mortality in patients with AMI. ${ }^{8-11}$ However, the patient can only benefit if correctly identified.

Although it is essential to identify all patients
Department of

Emergency Medicine, Accident and

Emergency,

Manchester Royal

Infirmary Oxford

Road, Manchester

M13 9WL, UK

Correspondence to: Kevin Mackway-Jones, Consultant

(kevin.mackway-jones@ man.ac.uk)

Accepted 5 October 2000 fer to coronary care, and also to limit the impact on the patient and healthcare resources.

The diagnosis of chest pains less than 12 hours in duration is an important challenge. This is for three reasons. Firstly, individual biochemical markers cannot effectively rule out myocardial infarction in the initial 12 hour period. ${ }^{13}{ }^{14}$ Secondly, aspirin and the fibrinolytic agents are at their most potent during this period, ${ }^{815}$ and finally the majority of AMI related deaths occur in the first 12 hours. ${ }^{4}$

Ideally a test would be available that identifies all AMIs immediately and confidently excludes all non-AMIs. No perfect test exists; instead tests are combined initially to rule in myocardial infarction (RIMI), and then to effectively rule out myocardial infarction (ROMI). The clinical efficacy of diagnostic tests is evaluated using sensitivity and specificity. To be certain of the diagnosis (in this case RIMI) a test must be have very few false positives (high specificity). However, to confidently test must have minimal false negatives (high sensitivity). ${ }^{16}$

The aim of this review is to discuss the evidence base underlying diagnostic and treatment strategies for patients with cardiac sounding chest pain.

\section{The initial approach to cardiac sounding chest pain}

Patients with cardiac sounding chest pain must have rapid access to appropriate care. This requires robust recognition of the problem, early ECG and assessment by a clinician trained to assess clinical risk. This is summarised in figure 1 .

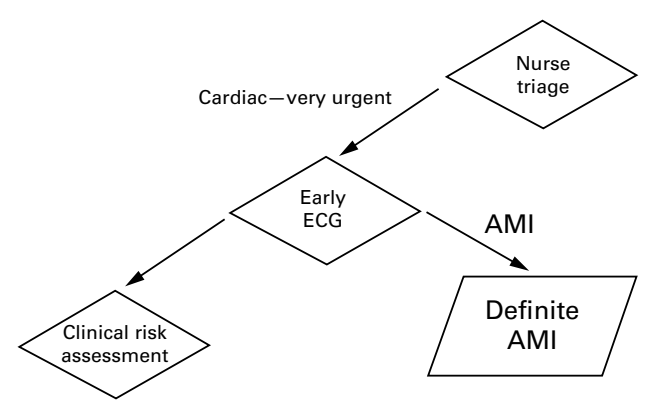

Figure 1 Initial approach. rule out a condition (in this case ROMI) the ust therefore be both timely and accurate order to facilitate early thrombolysis and trans- with AMI and unstable angina, it is also patients to unnecessary investigations, inpatient care and resultant psychological stress. Forty per cent of patients admitted to CCU with chest pain will have all ischaemic heart disease ruled out. ${ }^{12}$ The emotional, physical and economic impact on the patient, their family, their friends and the limited resources of the healthcare system should not be underestimated. The process of chest pain evaluation 
NURSE TRIAGE

The first clinical contact between the patient and the ED is usually at nurse triage. It is essential that cardiac sounding chest pain is identified at this stage, and accorded an appropriately high (very urgent) clinical priority. ${ }^{17}$ This will ensure that an appropriate early pathway of care is followed. Once this group of patients have been identified subsequent management should be presentation sensitivevery urgent cardiac pain patients should be placed in an appropriate area and ECG recording should be automatic.

\section{EARLY ECG}

The initial ECG is performed to RIMI, and should be recorded as soon as possible-and certainly within 10 minutes. The ECG is an excellent tool for RIMI as it is highly specific $(77 \%-100 \%)$ depending on the criteria used. However, the sensitivity of ECG is poor $(28 \%$ $54 \%$ ) in the first 12 hours, ${ }^{11} 18$ and the presence of a normal ECG neither excludes AMI nor provides sufficient assurance to discharge the patient from the ED. At this stage, therefore, the ECG is a tool to identify patients for consideration of fibrinolytic drugs. ${ }^{18-20}$

CLINICAL RISK STRATIFICATION

Acute MI patients with ECG changes should therefore be spotted straight away and should then be treated appropriately (see below). The patients who remain will range from those with unstable angina to those with musculoskeletal pain. While the particular diagnosis in individual patients may take some time to establish, the risks of either myocardial infarction or of later complications can be rapidly assessed by considering the ECG, by taking a focused history and by carrying out a brief examina-

Table 2 Clinical scenarios indicating a moderate risk of myocardial infarction in patients with normal ECGs

\begin{tabular}{ll}
\hline Scenario 1: & $\begin{array}{l}\text { Typical cardiac pain in a patient over } 40 \text { years old where the pain is not } \\
\text { reproduced by palpation, is not stabbing in nature and does not radiate } \\
\text { atypically. }\end{array}$ \\
Scenario 2: & $\begin{array}{l}\text { A history of anginal pain lasting longer than one hour that was either worse } \\
\text { than usual angina pain or as bad as the pain of a previous AMI. }\end{array}$ \\
\hline
\end{tabular}

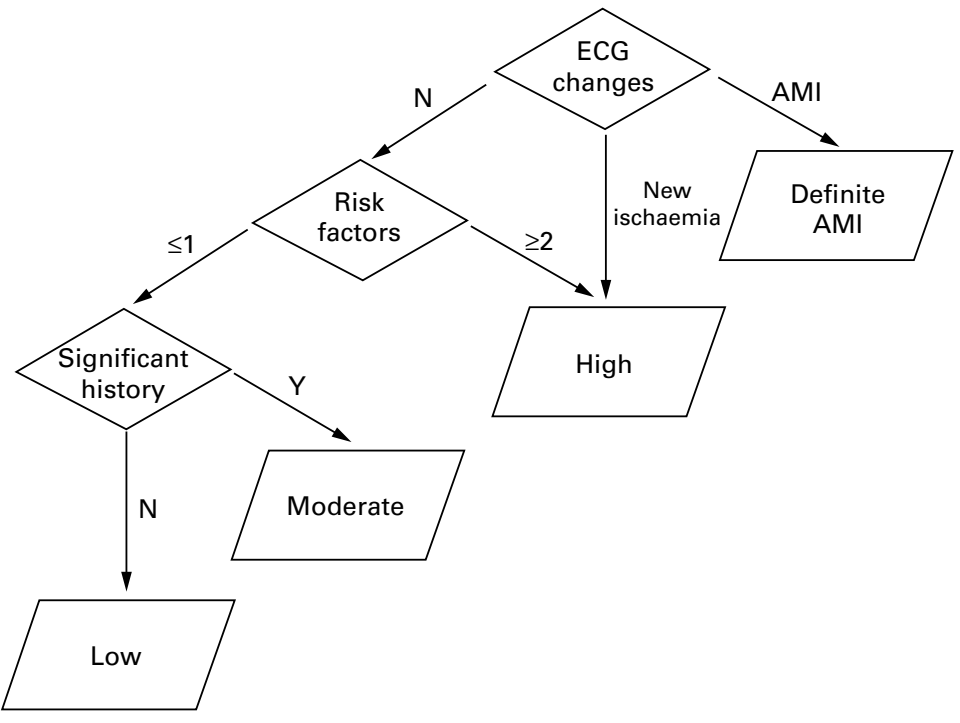

Figure 2 Clinical risk assessment overview.
Table 1 History suggesting unstable cardiac ischaemia

Cardiac sounding chest pain and any of:

- Pain the same as a previous AMI

- New onset of rest pain

- Pain not relieved by standard treatment in standard time

- Pain lasting more than 60 minutes

- Pain occurring with increasing frequency over the previous

24 hours

- Pain within six weeks of AMI or revascularisation

tion. This will allow appropriate decisions about further care to be made.

The ECG findings are considered firstischaemic changes not known to be old predict both a high risk of myocardial infarction and also a high risk of complications. If the ECG is normal then clinical risk factors are sought. Firstly, any history consistent with unstable ischaemic heart disease is elicited-a practical checklist is shown in table 1 .

Secondly, any findings of either hypotension (systolic blood pressure less than $120 \mathrm{~mm} \mathrm{Hg}$ ) or significant heart failure (crepitations not just including the bases) are noted. If more than two clinical risk factors are present then the patient is at high risk.

If only one risk factor is present or there are none at all, then the history should be reconsidered to see whether one of two particular scenarios that go along with a moderate risk of myocardial infarction are present. These are shown in table 2.

The whole approach to clinical risk assessment is summarised in figure 2 .

This assessment tool is derived from the multicentre chest pain study ${ }^{1920}$ and provides an objective, evidence based tool for use in the ED. It ensures AMI and other high risk patients are identified rapidly and provides a framework for subsequent care of all those remaining.

\section{Management}

The management of the patients will depend on the outcome of the initial screen. Some patients will have an ECG positive diagnosis of myocardial infarction and will need immediate intervention. Others will be at high risk and will need admission for both treatment and further diagnosis. Those at moderate and low risk will need myocardial infarction ruled out, and appropriate follow up arranged.

\section{DEFINITE AMI}

ST elevation ( $>1 \mathrm{~mm}$ in two limb leads or $>2$ $\mathrm{mm}$ in two chest leads) or acute left bundle branch block in a patient with chest pain are diagnostic of AMI and indicators for the use of fibrinolytic drugs. ${ }^{815}$

Patients should receive aspirin unless they have a major contraindication (active peptic ulceration, bleeding disorders and severe allergy). ${ }^{21}$ Aspirin inhibits cyclo-oxygenasedependent platelet activity - taking one hour to induce complete inhibition of cyclooxygenase. ${ }^{22}$ Therefore the earlier aspirin is given the greater the effect. Aspirin given immediately and continued for one month after AMI prevents 25 deaths and 13 other vascular events per 1000 patients treated..$^{10}$ 
Table 3 Contraindications to the use of fibrinolytic drugs

Recent haemorrhage, trauma or surgery

Bleeding disorders, for example, haemophilia, severe liver disease

History of bleeding, for example, cerebral bleed, GI bleed Severe hypertension $(>200 \mathrm{~mm} \mathrm{Hg}$ )

Cavitating lung disease

Acute pancreatitis

Table 4 Indications for the use of t-PA in patients with $S T$ segment elevation or acute $L B B B$

Previous streptokinase

Hypotension $(\mathrm{B}<110 \mathrm{~mm} \mathrm{Hg})$

Aged under 75

Anterior myocardial infarction

The two most commonly used fibrinolytics are streptokinase and recombinant tissue plasminogen activator (rtPA). Fibrinolytic agents work by direct action upon the coronary artery thrombosis leading to recanalisation and reperfusion of the myocardium supplied by the artery. ${ }^{23}$ Fibrinolytics and aspirin used in conjunction result in a reduction in mortality of 52 deaths per 1000 AMIs. ${ }^{10}$ The indications and contraindications for fibrinolytic agents are listed in tables 3 and 4 .

Individual hospitals have defined guidelines on the use of these drugs taking into account the cost differential and the relative clinical efficacy; currently rtPA costs up to eight times as much as streptokinase.

The benefit of thrombolysis is not age or sex dependent and fibrinolytics should be given to all indicated patients with no contraindications. The benefits are greatest in the sickest patients, those with hypotension and tachycardia. However, benefit is time dependent and rapid identification and initiation of treatment is essential. ${ }^{8}$

HIGH RISK GROUP

Patients at high risk of either myocardial infarction or complications will require emergency treatment and admission. Many of these patients have ST-T segment changes suggestive of myocardial ischaemia or subendocardial myocardial infarction, while others have a history strongly sugggestive of unstable ischaemic heart disease. This group has around a $25 \%$ chance of AMI, and a moderate to high risk of major complications developing. ${ }^{19} 20$

In common with all patients with possible cardiac chest pain they should receive aspirin and appropriate analgesia. $\beta$ Blockade should be started unless specifically contraindicated (by the presence of significant conduction disorder, definite asthma or overt heart failure) as should antithrombotic therapy. Low molecular weight heparin is more effective than unfractionated heparin at reducing the incidence of ischaemic events and the need for revascularisation procedures. The incidence of major bleeding complications is the same for both forms of heparin. Thus all patients who fall into the high risk chest pain group who are not eligible for fibrinolytic drugs should receive low molecular weight heparin. ${ }^{24}$
MODERATE RISK GROUP

The care of the moderate risk group is moot at present. They have a $7 \%-21 \%$ chance of having had an AMI and may either be managed by admission as high risk patients, or by entry into a ROMI protocol (see below).

LOW RISK GROUP

Some $65 \%$ of all patients presenting to an ED with cardiac sounding chest pain fall into the low risk group. These patients do not have a clear cut clinical diagnosis, the risk of AMI is less than $7 \%$ and the risk of a major complication is around $2 \%$. All patients should be screened for evidence of myocardial damage. ${ }^{1920}$

Traditionally some of these patients are admitted to hospital for a ROMI protocolnormally serial enzymes and ECGs-while others are discharged without further investigation. This approach results in a proportion of patients with AMI being inadvertently discharged. Matching the clinical resource with the clinical need has led to the development of chest pain assessment units (CPAUs). The purpose of these is twofold-firstly, to identify patients with myocardial damage rapidly and secondly, to facilitate discharge for the rest as quickly as safely possible. ${ }^{25-30}$ All this must be done cost effectively.

CURRENT STRATEGIES FOR ROMI

A variety of strategies currently exist for ROMI. These include cardiac enzyme assays, the ECG and other tests. The traditional approach of serial ECGs and enzyme testing (also known as the World Health Organisation criteria) is still the most commonly used ROMI and RIMI protocol. At 24 hours, and once two samples have been taken, the WHO criteria are $96 \%$ sensitive. ${ }^{31}$ In low risk patients in whom no further pain has occurred the sensitivity is $99.4 \% .^{30}$ This test is relatively cheap but not timely, requiring the patient to remain an inpatient for over 24 hours.

NEW BIOCHEMICAL TESTS

The traditional markers of creatinine kinase (CK), aspartate transaminase (AST) and lactate dehydrogenase $(\mathrm{LDH})$ are being superseded by newer tests. Entirely new markers such as the cardiac troponins have been developed, and new approaches to traditional enzymes have become available.

\section{Cardiac troponins}

The two new tests for troponins (cTnT and cTnI) are both highly specific and sensitive. Troponins are proteins that make up the tropomyosin regulatory complex (TRC). The TRC regulates the actin-myosin complex in muscle. Troponin $\mathrm{T}$ and I in cardiac muscle are unique in that they are virtually only produced by cardiac myocytes. ${ }^{32}$ The test identifies the amount of each protein in the blood. Testing for either TnT orTnI is both cost and clinically effective after 12 hours, but misses the early diagnostic window available to EDs. These enzymes are therefore better as late markers and not as ROMI tests in a six or nine 


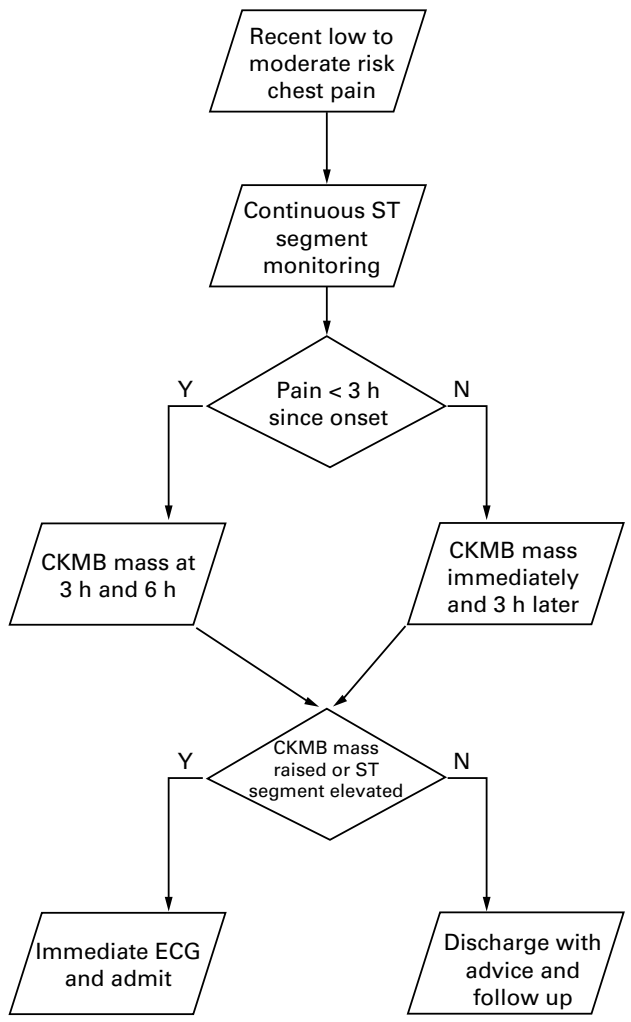

Figure 3 CPAU approach.

hour time window. The only study that evaluates cTnT in emergency department patients did not adequately evaluate discharged patients - the incidence of missed myocardial damage was not therefore assessed..$^{33}$

\section{$C K-M B$ isoforms and mass}

$\mathrm{CK}$ has three isoenzymes ( $\mathrm{MM}, \mathrm{BB}, \mathrm{MB}$ ). CK-MB has a higher cardiac specificity than $\mathrm{CK}$, and is a more sensitive and specific marker of myocardial damage. A number of isoforms of these enzymes exist. These isoforms are more sensitive and specific than CK but technically difficulties make their assay difficult to perform as an emergency service; furthermore the interpretation of the results (which are given as ratios) can be difficult. CK mass measurement is now possible - this measures the absolute amount of the enzyme - and is both easy to perform and interpret. Both CK isoforms and mass tests are specific and reasonably sensitive at six hours after onset of pain $(78 \%-100 \%)$. These tests are significantly cheaper than the cost of inpatient care, and so can be cost effective if they facilitate early safe discharge. ${ }^{14}{ }^{34-36}$

\section{Combining tests in CPAUs}

There are now multitudes of diagnostic tests to ROMI, some are early markers, some are cheaper than others are and some are more specific or sensitive. However, no single test will reliably ROMI in patients with fewer than 12 hours of chest pain. ${ }^{14}$

One way to increase sensitivity is to do a series of tests. This is the premise behind CPAUs-ED based ROMI protocols. The combination of CK-MB assay and ST segment monitoring has been extensively used in this way; a prospective randomised controlled trial has shown this approach to be safe, when compared with inpatient care. ${ }^{28}$

One approach is the six hour CPAU protocol shown in figure 3.

CPAU protocols can deliver high sensitivity and specificity and provide a rapid evidencebased protocol for ROMI in the ED. A negative test effectively rules out significant myocardial damage and allows safe discharge from the ED.

A number of other technologies and tests have been proposed as possible protocol additions for a chest pain service. These include troponin testing, exercise testing and echocardiography. ${ }^{36-38}$ The main problem at present in including these technologies in ED protocols is that they are not validated for use in patients with the same spectrum of disease as ED patients

\section{Summary}

All patients attending an ED with chest pain that could be cardiac should be given a high triage priority to allow rapid assessment and treatment.

All patients should receive adequate analgesia and aspirin. Patients with AMI who require fibrinolytic agents should be identified and treatment started. Other high risk patients need inpatient care and may need low molecular weight heparin.

Low risk patients require rapid, cost effective and efficacious ROMI protocols, so they can be discharged safely. CPAUs provide the best way of achieving this. Currently the best early protocol seems to be serial CK-MB measurements and continuous ST segment monitoring

1 Fothergill NJ, Hunt MT, Touquet R. Audit of patients with chest pain presenting to an accident and emergency department over a 6-month period. Arch Emerg Med 1993;10:155-60.

2 Emerson PA, Russell NJ, Wyatt J, et al. An audit of doctors management of patients with chest pain in the accident and emergency department. Qf Med 1989;70:213-20.

3 Mortality statistics. Series DH2. London: HMSO, 1996:23.

Norris RM. On behalf of the United Kingdom heart attack study collaborative group. Fatality outside hospital from acute coronary events in three British health districts 1994-5. BMF 1998;316:1065-70.

5 Collinson PO, Premachandram S, Hashemi K. Prospective audit of incidence of prognostically important myocardial damage in patients discharged from emergency department. BMF 2000;320:1702-5

6 Lee TH, Rouan GW, Weisberg M, et al. Clinical characteristics and natural history of patients with acute myocardial infarction sent home from the emergency department. Am 7 Cardiol 1987;60:219-24.

7 Karcz A, Holbrook J, Burke MC, et al. Massachusetts Emergency Medicine closed malpractice claims:1988-90. Ann Emerg Med 1993;22:553-9.

8 Fibrinolytic Therapy Trialists collaborative group. Indications for fibrinolytic therapy in suspected acute myocardial infarction: collaborative overview of early mortality and infarction: collaborative overview of early mortality and major morbidity results from all major randomised tha

9 ISIS-1 (First international study of infarct survival) collaborative group. Randomised trial of intravenous atenolol among 16027 cases of suspected acute myocardial infarction:ISIS-1. Lancet 1986;ii:57-66.

10 ISIS-2 (Second international study of infarct survival) collaborative group. ISIS-3: a randomised comparison of streptokinase vs tissue plasminogen activator vs anistreplase and of aspirin plus heparin vs aspirin alone in 41299 cases of suspected acute myocardial infarction. Lancet 1992;339:753-70.

11 King III SB, Lembo NJ, Weintraub WS, et al. A randomised controlled trial comparing coronary angioplasty with 2 Schroeder JS, Lamb IH, Harrison DC. Patients admitted to the coronary care unit for chest pain: High risk subgroup for subsequent cardiovascular death. Am f Cardiol 1977;39: $829-32$. 
13 Young GP, Green TR. The role of single ECG, creatine kinase, and CKMB in kinase, and CKMB in diagnosing patie

14 Bakker AJ, Koelemay MJW, Gorgels PMC, et al. Failure of new biochemical markers to exclude acute myocardial infarction at admission. Lancet 1993;342:1220-2.

15 GUSTO investigators. An international randomised trial comparing four thrombolytic strategies for acute myocardial infarction. N Engl F Med 1993;329:673-2.

16 Sackett DL, Haynes RB, Guyatt GH, et al. Clinical epidemiology: a basic science for clinical epidemiology. 2nd ed. London: Little, Brown, 1991.

17 Mackway-Jones K, ed. Emergency triage. London: BMJ Publishing, 1997

18 Brush JE, Brand DA, Acampora D, et al. Use of the initial electrocardiogram to predict in hospital complications of acute myocardial infarction. N Engl f Med 1985;312:113741 .

19 Goldman L, Cook EF, Brand DA, et al. A computer protocol to predict myocardial infarction in emergency department to predict myocardial infarction in emergency department

20 Goldman L, Cook EF, Johnson PA, et al. Prediction of the need for intensive care in patients who come to emergency
departments with acute chest pain. $N$ Engl $\mathcal{f}^{\mathrm{Med}}$ departments with acu

21 British National Formulary. 33rd ed. London: British Medical Association and Royal Pharmaceutical Society of Great Britain, 1999 .

22 Clarke RJ, Mayo G, Price P, et al. Suppression of thromboxane A2 but not of systemic prostacyclin by controlled release aspirin. $N$ Engl f Med 1991;325:1137-41.

23 Schoder R, Biamono G, Von Leitner ER. Intravenous short term infusion of streptokinase in acute myocardial infarction. Circulation 1983;67:536-48.

24 Cohen M, Demers C, Gurfinkel EP, et al. A comparision of low molecular weight heparin with unfractionated heparin for unstable coronary artery disease. $N$ Engl $\mathcal{f}$ Med for unstable cor

25 Gibler WB, Runyon JP, Levy RC, et al. A rapid diagnostic protocol and treatment centre for patients with chest pain in protocol and treatment centre for patients with chest pain

26 Gaspoz JM, Lee TH, Cook EF, et al. Outcome of patients Gaspoz JM, Lee TH, Cook EF, et al. Outcome of patients
who were admitted to a new short stay unit to rule out who were admitted to a new short stay unit to rule
myocardial infarction. Am 7 Cardiol 1991;68:145-9.

27 De Leon AC, Farmer CA, King G, et al. Chest pain evaluation unit: A cost effective approach for ruling out acute myocardial infarction. Scott Med $\mathcal{F} 1989 ; 82: 1083-9$.
28 Zalenski RJ, McCarren M, Roberts R, et al. An evaluation of a chest pain diagnostic protocol to exclude acute cardiac ischaemia in the emergency department. Arch Intern Med 1997;157:1085-91.

29 Farkouh ME, Smars PA, Reeder GS, et al. A clinical trial of a chest pain observation unit for patients with unstable angina. $N$ Engl F Med 1998;339:1882-8.

30 Lee TH, Juarez GJ, Cook EF, et al. Ruling out myocardial infarction: A prospective multicenter validation of a 12 hour strategy for patients at low risk. $N \mathrm{Engl} \mathrm{f} \mathrm{Med}$ 1996;334:1498-504

31 Lee TH, Rouan GW, Weisberg M, et al. Sensitivity of routine clinical criteria for diagnosing myocardial infarction within 24 hours of hospitalisation. Ann Intern Med 1987;106:181-6.

32 Mair J. Progress in myocardial damage detection: new biochemical markers for clinicians. Crit Rev Lab Sci 1997; 34:1-66.

33 Hamm CW, Goldmann BU, Heeschen C, et al. Emergency room triage of patients with acute chest pain by means of rapid testing for cardiac troponin T or troponin I. N Engl f Med 1997;337:1648-53.

34 Young GP, Gibler WB, Hedges JR, et al. Serial creatinine kinase-MB results are a sensitive indicator of acute myocardial infarction in chest pain patients with non diagnostic electrocardiograms: The second emergency medicine cardiac research group study. Acad Emerg Med 1997;4: 869-77

35 Mair J, Morandell D, Genser N, et al. Equivalent early sensitivities of myoglobin, creatinine kinase $\mathrm{MB}$ mass, creatinine kinase isoform ratios and cardiac troponins I and $T$ for acute myocardial infarction. Clin Chem 1995;41: 1266-72.

36 Leung FY, Griffith AP, Jablonsky G, et al. Comparison of the diagnostic utility of timed serial (slope) creatinine kinase measurements with conventional serum tests in the early diagnosis of myocardial infarction. Ann Clin Biochem 1991; 28:78-82.

37 Lindsey J Jr, Bonnet YD, Pinnow EE. Routine stress testing for triage of patients with chest pain: Is it worth the candle? Ann Emerg Med 1998;32:600-3.

38 Lewis WR, Amsterdam EA. Evaluation of the patient with rule out myocardial infarction. Arch Intern Med 1996;156: $41-5$ 